TRANSACTIONS OF THE

AMERICAN MATHEMATICAL SOCIETY

Volume 359, Number 8, August 2007, Pages 3567-3575

S 0002-9947(07)04104-9

Article electronically published on March 7, 2007

\title{
EIGENVALUE ESTIMATES FOR MINIMAL SURFACES IN HYPERBOLIC SPACE
}

\author{
ALBERTO CANDEL
}

\begin{abstract}
This paper gives an upper bound for the first eigenvalue of the universal cover of a complete, stable minimal surface in hyperbolic space, and a sharper one for least area disks.
\end{abstract}

\section{INTRODUCTION}

Estimates for the eigenvalues of minimal surfaces in hyperbolic space, and in space forms in general, have been obtained using comparison methods by Cheng, $\mathrm{Li}$ and $\mathrm{Yau}$ [8, for instance.

The purpose of this paper is to give an explicit upper bound for the first eigenvalue of certain classes of immersed minimal surfaces in hyperbolic space. One such class consists of those minimal surfaces which satisfy the stability condition for the second variation of area.

The other class of minimal surfaces for which an explicit upper bound for the first eigenvalue can be obtained is the class of proper, least area disks. These surfaces are in particular stable minimal surfaces, but their geometric structure can be used to obtain a shaper upper bound for their first eigenvalue.

Schoen [18 proved that the curvature of an immersed stable minimal surface in a three manifold at a point in the surface is bounded in terms of the injectivity radius at that point. In particular, if the immersion is complete, then the curvature is bounded. This immediately implies the existence of upper and lower bounds for the first eigenvalue by standard comparison theorems. It is possible, in the case that the ambient space is hyperbolic space, to obtain actual numerical values for the bounds on the curvature of stable minimal surfaces, and thus for their first eigenvalues, by tracing through the proof of Schoen's theorem, but the results that I was able to obtain in that way are not as optimal as those proved below.

Another approach to obtaining bounds for the curvature, and thus for the eigenvalue, of a minimal surface could use the Weierstrass representation of minimal surfaces in hyperbolic space obtained by Kokubu [13, but this will not be discussed here.

\section{Minimal surfaces in hyperbolic space}

This section contains some generalities pertaining to minimal surfaces in hyperbolic space, and to eigenvalues of Riemannian manifolds. Most of them are

Received by the editors February 14, 2005.

2000 Mathematics Subject Classification. Primary 53A10, 53C21.

This research was supported by N.S.F. Grant 0205825 .

(C)2007 American Mathematical Society Reverts to public domain 28 years from publication 
known or are otherwise easy to derive, but are included here to fix notation and for convenience of the reader, as they will be invoked in later sections.

Let $\mathbf{H}^{3}$ denote hyperbolic space of constant curvature -1 . If $j: F \rightarrow \mathbf{H}^{3}$ is an isometric immersion of a surface $F$ in hyperbolic space, then the second fundamental form of $F$ is the bilinear form $B: T F \otimes T F \rightarrow T^{\perp} F$ with values in the normal bundle to $F$ given by

$$
B(X, Y)=\tilde{\nabla}_{X} Y-\nabla_{X} Y
$$

where $\tilde{\nabla}$ and $\nabla$ are the connections of $\mathbf{H}^{3}$ and $F$, respectively.

By definition, an immersion $j: F \rightarrow \mathbf{H}^{3}$ is called a minimal immersion (or $F$ is said to be a minimal surface, for short) if its second fundamental form has trace identically equal to 0 .

It follows that if $\tilde{f}$ is a smooth function on $\mathbf{H}^{3}$ (or on a portion thereof), and if $f$ denotes the induced function on $F$, then, on $F$, their respective Hessians are related by

$$
\tilde{\nabla}_{X, Y}^{2} \tilde{f}-\nabla_{X, Y}^{2} f=\langle B(X, Y), \tilde{\nabla} \tilde{f}\rangle,
$$

for any $X$ and $Y$ tangent vector fields to $F$. Therefore, if $F$ is a minimal surface, the Laplace operators on $\mathbf{H}^{3}$ and on $F$ of the functions $\tilde{f}$ and $f$, respectively, are related by

$$
\tilde{\Delta} \tilde{f}-\Delta f=\tilde{\nabla}_{N, N}^{2} \tilde{f}
$$

where $N$ is the positively oriented unit normal vector to $F$.

The term on the right side of the above expression can be computed when the function $\tilde{f}$ is a distance function. That is, let $\tilde{r}$ be the function on $\mathbf{H}^{3}$ given by the distance to a fixed point in $\mathbf{H}^{3}$. Then the Hessian of $\tilde{r}$ satisfies the identity

$$
\tilde{\nabla}_{X, Y}^{2} \tilde{r}=(\langle X, Y\rangle-\langle X, \tilde{\nabla} r\rangle\langle Y, \tilde{\nabla} r\rangle) \operatorname{coth} \tilde{r} .
$$

This follows by a lengthy but otherwise straightforward calculation in polar coordinates in $\mathbf{H}^{3}$ centered at the point that was used to determine $\tilde{r}$.

It follows from this that the Laplacian of $\tilde{r}$ satisfies the identity $\tilde{\Delta} \tilde{r}=2 \operatorname{coth} \tilde{r}$, and thus that the Laplacian of $r$, the distance function on $F$ induced by $\tilde{r}$, satisfies the equation

$$
\Delta r=\left(2-|\nabla r|^{2}\right) \operatorname{coth} r
$$

One immediate consequence of this identity is that minimal surfaces in $\mathbf{H}^{3}$ satisfy a linear isoperimetric inequality. Indeed, if $F \rightarrow \mathbf{H}^{3}$ is a minimal surface and $D \subset F$ is a relatively compact domain with smooth boundary and outward normal vector $\nu$, then integration by parts yields

$$
\text { Area } D \leq \int_{D} \Delta r=\int_{\partial D}\langle\nabla r, \nu\rangle \leq \text { Length } \partial D,
$$

because $\Delta r=\left(2-|\nabla r|^{2}\right) \operatorname{coth} r \geq 1$ and $|\nabla r| \leq 1$. (Sharper isoperimetric inequalities and Sobolev-type inequalities for minimal surfaces in space forms were obtained by Choe and Gulliver 9 .)

Definition 1. The first eigenvalue of a Riemannian manifold, $M$, is given by the Rayleigh quotient

$$
\lambda(M)=\inf _{f} \frac{\int_{M}|\nabla f|^{2}}{\int_{M} f^{2}},
$$

where the infimum is taken over all compactly supported Lipschitz functions on the manifold $M$. 
One property of the first eigenvalue of a Riemannian manifold which will be used below is that it satisfies the identity

$$
\lambda=\inf _{D} \lambda(D)
$$

where the infimum is taken over all relatively compact domains in the manifold.

The isoperimetric inequality (1) says that the isoperimetric constant of a complete minimal surface in hyperbolic space is $\geq 1$, and thus Cheeger's inequality for non-compact complete Riemannian manifolds (cf. [7, Theorem VI.1.2]) implies that the first eigenvalue of such a surface is $\geq \frac{1}{4}$. In the next two sections, upper bounds for $\lambda$ will be obtained under certain geometric conditions.

\section{Stable minimal SURfaces AND the First EIGENVAlue}

The purpose of this section is to obtain upper bounds for the first eigenvalue of stable minimal surfaces, which are defined as follows.

Definition 2. A minimal surface is stable if the second variational formula for the area functional on compactly supported normal deformations is non-negative.

Analytically this means that if $F \rightarrow \mathbf{H}^{3}$ is a minimal isometric immersion, then $F$ is stable if and only if

$$
\int_{F}\left(|B|^{2}-2\right) f^{2} \leq \int_{F}|\nabla f|^{2}
$$

for all compactly supported Lipschitz functions $f$ on $F$.

The curvature, $K$, and the norm of the second fundamental form, $|B|$, of a minimal surface in hyperbolic space satisfy the following relation (Gauss' lemma):

$$
K=-1-\frac{|B|^{2}}{2} \text {. }
$$

Therefore, the stability inequality (4) can be written in the form

$$
0 \leq 2 \int K f^{2}+4 \int f^{2}+\int|\nabla f|^{2},
$$

for any compactly supported function $f$ on $F$.

As noted in Section 2, the first eigenvalue of a complete minimal surface in hyperbolic space is bounded below by $\frac{1}{4}$. The following theorem gives an upper bound when such a surface is also stable and simply connected.

Theorem 1. Let $F \rightarrow \mathbf{H}^{3}$ be a stable minimal immersion of a simply connected surface $F$ in hyperbolic space. Then the first eigenvalue of $F$ satisfies

$$
\frac{1}{4} \leq \lambda(F) \leq \frac{4}{3} .
$$

If the surface was not simply connected but still stable, then its universal cover would also be stable; this is proved in do Carmo and Peng [6, Lemma 1]. Therefore Theorem 1 applies to the universal cover of any complete stable minimal surface in hyperbolic space.

Proof. The starting point to find an upper bound for the first eigenvalue of the surface $F \rightarrow \mathbf{H}^{3}$ in the statement of Theorem 1 is to write down the stability inequality (44) in polar coordinates. This method was used by Pogorelov [16] in 
his proof that stable minimal surfaces in Euclidean space are planes (a result first obtained by do Carmo and Peng [6] and by Fisher-Colbrie and Schoen [10]).

Thus because $F$ is simply connected and has negative curvature, there are global polar coordinates about any point in $F$. Under such coordinates, the metric tensor of $F$ can be written in the form

$$
d r^{2}+g(r, \theta)^{2} d \theta^{2}
$$

where the function $g$ satisfies

$$
g(0, \theta)=0 \quad \text { and } \quad g_{r}^{\prime}(0, \theta)=1 .
$$

The curvature, $K$, of $F$ is then given by $K=-\frac{g_{r r}^{\prime \prime}}{g}$.

Consider compactly supported functions on $F$ which depend only on the distance $r$ to the origin of the polar coordinate system in $F$ and are piecewise linear in $r$. The claim is now that if $f$ is any of those functions, then the stability condition (5) takes on the following form:

$$
4 \pi f(0)^{2}+4 \int_{F} f^{2} \geq 3 \int_{F}|\nabla f|^{2} .
$$

Indeed, if $f$ is one of such functions with $f(r)=0$ for $r \geq R$, the stability condition in the polar coordinates $(r, \theta)$ can be written in the form

$$
\int_{0}^{2 \pi} \int_{0}^{R}\left(f_{r}^{\prime}\right)^{2} g d r d \theta+4 \int_{0}^{2 \pi} \int_{0}^{R} f^{2} g d r d \theta \geq 2 \int_{0}^{2 \pi} \int_{0}^{R} f^{2} g_{r r}^{\prime \prime} d r d \theta
$$

where the fact that $-K g=g_{r r}^{\prime \prime}$ was used. Integrate the right side of the above expression twice by parts to obtain that

$$
\begin{aligned}
\int_{0}^{R} f^{2} g_{r r}^{\prime \prime} d r & =\left.f^{2} g_{r}^{\prime}\right|_{0} ^{R}-\int_{0}^{R} 2 f f^{\prime} g_{r}^{\prime} \\
& =-f(0)^{2}+\int_{0}^{R} 2\left(\left(f^{\prime}\right)^{2}+f f^{\prime \prime}\right) g \\
& =-f(0)^{2}+2 \int_{0}^{R}\left(f^{\prime}\right)^{2} g .
\end{aligned}
$$

The second equality is because $f(R)=0, g^{\prime}(0, \theta)=1$, and $g(0)=0$; the third identity is because $f$ is piecewise linear in $r$, that is, $f^{\prime \prime}(r)=0$ except for finitely many values of $r$. Hence

$$
2 \int K f^{2}=4 \pi f^{2}(0)-4 \int|\nabla f|^{2}
$$

and (6) follows by plugging this into (5).

Let $\mathcal{F}$ be the family of radial piecewise linear functions on $F$ with $f(0)=0$. The stability condition in the form (66) implies that if $f \in \mathcal{F}$, then

$$
4 \int f^{2} \geq 3 \int|\nabla f|^{2}
$$

and thus that

$$
\lambda(F) \leq \inf _{f \in \mathcal{F}} \frac{\int|\nabla f|^{2}}{\int f^{2}} \leq \frac{4}{3},
$$

completing the proof of Theorem 1 . 
Pogorelov's method of studying the stability inequality in polar coordinates was also used by Kawai [12] for estimating positivity of Schrödinger operators on surfaces related to the stability operator. In fact, a family of test functions similar to those used by Kawai can be used here to estimate the growth of a stable minimal surface in hyperbolic space. This is done as follows.

First define the (lower) growth rate, $\rho$, of a complete Riemannian manifold by

$$
\rho=\liminf _{r \rightarrow \infty} \frac{1}{r} \log \operatorname{Vol} B\left(x_{0}, r\right)
$$

where $x_{0}$ is an arbitrary point in the manifold (this definition of $\rho$ is independent of the point $x_{0}$ ).

Let $F$ be a minimal surface as in Theorem 1, Using polar coordinates on $F$, the length of the circle of radius $r$ about the point of reference 0 (that is, the length of the boundary of $B(0, r))$ is given by

$$
\text { Length } \partial B(0, r)=\int_{0}^{2 \pi} g(r, \theta) d \theta \text {. }
$$

Let $f_{R}(r)=(1-r / R)^{a R}$ for $r \leq R$ and 0 for $r \geq R$. Then, as $R \rightarrow \infty, f_{R}(r)$ converges uniformly on compact sets to the exponential function $\exp (-a r)$.

The stability condition (6) applied to the function $f_{R}$ takes on the form

$$
\begin{aligned}
4 \pi & \geq \int_{0}^{R}\left(3 a^{2}+\frac{4 a(a R-1)}{R^{2}}-4\left(1-\frac{r}{R}\right)^{2}\right)\left(1-\frac{r}{R}\right)^{2 a R-2} \text { Length } \partial B(0, r) d r \\
& \geq \int_{0}^{R}\left(3 a^{2}-4+\frac{4 a(a R-1)}{R^{2}}\right)\left(1-\frac{r}{R}\right)^{2 a R-2} \text { Length } \partial B(0, r) d r .
\end{aligned}
$$

If $a>\frac{2}{\sqrt{3}}$, then the integrand is positive (for $R$ is sufficiently large), and taking the limit when $R \rightarrow \infty$ it obtains that

$$
0 \leq \int_{0}^{\infty} \frac{\text { Length } \partial B(0, r)}{e^{2 a r}} \leq \frac{4 \pi}{3 a^{2}-4}<\infty .
$$

Therefore, $\liminf _{r \rightarrow \infty} \frac{\text { Length } \partial B(0, r)}{e^{2 a r}}=0$ if $a>2 / \sqrt{3}$. Because of the isoperimetric inequality for a minimal surface as derived in Section 2,

$$
\text { Area } B(0, r) \leq \text { Length } \partial B(0, r),
$$

and so the following statement has been proved.

Theorem 2. If $F \rightarrow \mathbf{H}^{3}$ is a complete, stable, isometric minimal immersion of a simply connected surface into hyperbolic space, then the (lower) growth rate of $F$ satisfies $\rho \leq \frac{4}{\sqrt{3}}$.

Relationships between stability, the first eigenvalue of the Laplacian, and other geometric properties of minimal surfaces have been explored in depth by Barbosa and do Carmo 3 and Mori [14. One basic relationship arises in the following form: a complete minimal surface in hyperbolic space is stable if $|B| \leq \frac{3}{2}$. In this case the curvature of the surface is $\geq-\frac{9}{4}$ and so the growth rate is $\leq \sqrt{\frac{17}{8}}$, as it follows by standard comparison arguments. Theorem 2 is a somewhat weak converse to this fact, giving a growth rate bound without having a curvature bound. 


\section{The FIRST EIGENVALUE OF A LEAST AREA DISK}

The theorem in this section gives an upper bound for the first eigenvalue of a least area disk in hyperbolic space. These are examples of minimal surfaces, and are defined as follows.

Definition 3. An (isometric) embedding $j: F \rightarrow \mathbf{H}^{3}$ of a non-compact, simply connected surface $F$ into $\mathbf{H}^{3}$ is called a least area disk if it is homotopically areaminimizing in the following sense: if $D \subset F$ is a compact disk in $F$ and $f: F \rightarrow \mathbf{H}^{3}$ is a mapping such that $f=j$ on $F \backslash D$, then Area $j(D) \leq$ Area $f(D)$.

It is apparent that least area disks are stable minimal surfaces, so Theorem 1 applies to them. However their peculiar geometric structure permits us to improve that bound. This requires the following definition.

Definition 4. The (upper) growth rate of a complete Riemannian manifold is the number $\mu$ defined by

$$
\mu=\limsup _{r \rightarrow \infty} \frac{1}{r} \log \operatorname{Vol} B\left(x_{0}, r\right) .
$$

Brooks 44 proved that the essential spectrum, $\lambda^{\text {ess }}$, of a complete Riemannian manifold of infinite volume satisfies $\lambda^{\text {ess }} \leq \frac{\mu^{2}}{4}$. Because the first eigenvalue, $\lambda$, always satisfies $\lambda \leq \lambda^{\text {ess }}$, this result of Brooks will now be used to obtain an upper estimate for the first eigenvalue of a complete, proper, least area disk in hyperbolic space. Some notation and a lemma are required.

Let $F \rightarrow \mathbf{H}^{3}$ be a proper minimal immersion of a surface $F$ into $\mathbf{H}^{3}$. Let $p$ be a point in $F$ and let $r$ be the distance function from $p$ induced on $F$. For each $s \geq 0$, let $F(p, s)$ denote a connected component containing $p$ of the set of points $x$ in $F$ such that $r(x)<s$. This set is relatively compact because $F$ is proper.

Lemma 1. Let $F \rightarrow \mathbf{H}^{3}$ be a proper minimal immersions of a simply connected surface. Then $F(p, R)$ is a disk for all $R>0$.

Proof. It was shown in Section 2 that the distance function on $F$ satisfies the differential inequality $\Delta r \geq 1$. In particular, $r$ has no local maxima. The distance function $r$ is smooth on $F \backslash\{p\}$, and so Sard's theorem implies that almost all its values are regular values. Thus, for almost all $s$, the level sets are disjoint unions of circles. If, for some value of $s$, two of those circles were nested, then the complement of $\{r=s\}$ would be a compact component. In this case, the function $r$ would have a local maximum, a contradiction to what was said at the beginning of this proof.

Theorem 3. Let $F \rightarrow \mathbf{H}^{3}$ be a proper least area disk. Then the first eigenvalue, $\lambda$, of $F \mathbf{H}^{3}$ satisfies

$$
\frac{1}{4} \leq \lambda \leq 1
$$

Proof. Let $r>0$ be such that $F(p, r)$ is a disk in $F$ (cf. Lemma 1), and let $D$ be its closure in $F$. Then, because $j$ is an embedding, $j(\partial D)$ is a simple closed curve in the sphere of radius $r$ and center $j(p)$ in $\mathbf{H}^{3}$. That curve divides this sphere into two disks, and thus one of those disks has area at most half the area of that sphere, which is $2 \pi \sinh ^{2}(r)$. Because $F$ is a least area disk, the area of $F(p, r)$ is then at most half that of the boundary of the geodesic ball in $\mathbf{H}^{3}$ of radius $r$ and center $p$, that is

$$
\text { Area } F(p, r) \leq 2 \pi \sinh ^{2}(r)
$$


Because $B_{F}(p, r)$, the geodesic disk in $F$ of radius $r$ and center $p$, is connected, $B_{F}(p, r) \subset F(p, r)$, and thus the upper growth rate $\mu$ of $F$ satisfies $\mu \leq 2$. Because of the theorem of Brooks mentioned at the beginning of this section, this implies that the first eigenvalue, $\lambda$, of $F$ satisfies $\lambda \leq 1$, as advertised in Theorem 3 .

\section{EXAMPLES AND COMMENTS}

It would be interesting to determine if the eigenvalue bounds established in Theorems 1 and 3 are sharp. The lower bound is sharp (totally geodesic disks), but the sharpness of the upper bound seems difficult but quite likely true because there are a multitude of examples of isometric stable minimal immersions of a simple connected surface into hyperbolic space (unlike the situation in Euclidean space, where all such surfaces are planes). These constructions arise from the work of Anderson [1] more examples were given by Polthier [17] and by Oliveira and Soret [15]. A general theorem of Uhlenbeck [19] establishes that given a quadratic differential on the hyperbolic plane with norm less than a given constant and a metric satisfying a certain partial differential equation, there is a stable minimal immersion of the hyperbolic plane into a hyperbolic space whose second fundamental form is the given quadratic differential. However, determining the constant involved in that theorem of Uhlenbeck and solving the differential equation does not appear to be straightforward.

Examples of minimal surfaces where explicit calculations can be done were given in do Carmo and Drajzer [5]. These generalize to a hyperbolic space the well-known examples of the cathenoids in Euclidean space. Two such families of examples, the hyperbolic and the parabolic cathenoids, have curvature bounded below by -2 , and therefore their first eigenvalue satisfies $\lambda \leq \frac{1}{2}$. These examples are also proper least area disks (cf. below). The other family of examples, the spherical cathenoids, are not proper because their image in hyperbolic space is a cylinder.

Theorem 4. The first eigenvalue of a cathenoid in hyperbolic space is $\lambda=\frac{1}{4}$.

Proof. The metric on the cathenoids has the expression (derived in [5])

$$
d s^{2}+(a \cosh 2 s-\delta / 2) d t^{2},
$$

in the plane $-\infty<s, t<\infty$, where $\delta$ takes on the values $-1,0,1$, and where $a>1 / 2$ if $\delta \neq 0$ and $a=1$ if $\delta=0$.

Assume that $\delta=-1$ (the other cases are similar). Let $\mathbf{H}^{2}$ denote the hyperbolic plane and write its metric in polar coordinates: $d r^{2}+2 a \sinh ^{2} r d \theta^{2}$, with $0 \leq r<\infty$ and $0 \leq \theta \leq 2 \pi / \sqrt{2 a}$. Let $H_{r_{0}}$ be the region in $\mathbf{H}^{2}$ consisting of points with coordinates $r>r_{0}$ and $0<\theta<2 \pi / \sqrt{2 a}$. Then the mapping $\phi(r, \theta)=(r, \theta)$ from the region $H_{0}$ into $F$ has norm

$$
1-A(r) \leq|d \phi|^{2} \leq 1
$$

Here $A(r)=4 \operatorname{csch} 2 r$, so $A(r) \rightarrow 0$ as $r \rightarrow \infty$.

If $D$ is a geodesic disk in that region $H_{0}$ of $\mathbf{H}^{2}$ and $D_{F}$ is the disk in $F$ corresponding to $D$ under $\phi$, then the Rayleigh quotient definition (2) of $\lambda$ immediately gives the following comparison for the eigenvalues of $D$ and $D_{F}$ :

$$
(1-A(r))^{2} \lambda(D) \leq \lambda\left(D_{F}\right) \leq \frac{1}{(1-A(r))^{2}} \lambda(D) .
$$


For any $r_{0}>0$, the region $H_{r_{0}}$ in $\mathbf{H}^{2}$ contains geodesic disks of arbitrarily large radius. Because the first eigenvalue of a non-compact manifold satisfies (3), and because $\lambda\left(\mathbf{H}^{2}\right)=\frac{1}{4}$, there is a geodesic disk $D$ in that region of $\mathbf{H}^{2}$ such that $\lambda(D)$ is as close to $\frac{1}{4}$ as desired. By homogeneity of $\mathbf{H}^{2}$, this disk $D$ can be assumed to be contained in any region $H_{r_{0}}$, with $r_{0}$ arbitrarily large. It then follows from (7) that the corresponding relatively compact domain $D_{F}$ in $F$ has $\lambda\left(D_{F}\right)$ as close to $\frac{1}{4}$ as desired, and so the proof is complete because of (3).

The hyperbolic $(\delta=-1)$ and parabolic $(\delta=0)$ cathenoids are not only stable minimal embeddings of the plane into $\mathbf{H}^{3}$, but are in fact properly embedded least area disks. This is proved as follows. By a calculation done in [5], the norm of the second fundamental form of these minimal surfaces is $\leq \sqrt{2}$. Then the proof of Theorem 3 in Uhlenbeck [19] can be easily adapted to the present situation (it is stated for compact surfaces) to show that such surfaces are in fact homologically least area, hence least area disks.

To conclude, note that, in general, a proper least area disk need not have second fundamental form bounded above by $\sqrt{2}$ everywhere (equivalently, curvature bounded below by -2 ). This is so because if a complete minimal immersion of a surface $F$ with curvature $K \geq-2$, then its exterior curvature is $\leq 0$, and thus the exponential map from the normal bundle to $F$ into $\mathbf{H}^{3}$ is a diffeomorphism (by the Cartan-Hadamard theorem as generalized in Hermann [11, or by an explicit calculation done in [19]). This property of the exponential map implies that the intersection of such a surface with every geodesic ball in $\mathbf{H}^{3}$ is connected. By using the convex hull property for minimal surfaces in hyperbolic space (cf. Anderson [1]), it is not difficult to construct a least area disk in $\mathbf{H}^{3}$ whose intersection with some geodesic ball is not connected. Indeed, take an appropriate "tennis ball" curve in the visual boundary of a hyperbolic space and apply the existence theorems for least area disks with boundary a given curve at infinity as given in Anderson [2].

\section{REFERENCES}

[1] Michael T. Anderson, Complete minimal varieties in hyperbolic space, Invent. Math. 69 (1982), no. 3, 477-494. MR0679768 (84c:53005)

[2] Complete minimal hypersurfaces in hyperbolic n-manifolds, Comment. Math. Helv. 58 (1983), no. 2, 264-290. MR0705537 (85e:53076)

[3] João Lucas Barbosa and Manfredo do Carmo, Stability of minimal surfaces and eigenvalues of the Laplacian, Math. Z. 173 (1980), no. 1, 13-28. MR0584346 (81h:53007)

[4] Robert Brooks, A relation between growth and the spectrum of the Laplacian, Math. Z. 178 (1981), no. 4, 501-508. MR0638814 (83a:58089)

[5] Manfredo do Carmo and M. Dajczer, Rotation hypersurfaces in spaces of constant curvature, Trans. Amer. Math. Soc. 277 (1983), no. 2, 685-709. MR0694383 (85b:53055)

[6] Manfredo do Carmo and C. K. Peng, Stable complete minimal surfaces in $\mathbf{R}^{3}$ are planes, Bull. Amer. Math. Soc. (N.S.) 1 (1979), no. 6, 903-906. MR0546314 (80j:53012)

[7] Isaac Chavel, Isoperimetric inequalities, Cambridge Tracts in Mathematics, vol. 145, Cambridge University Press, Cambridge, 2001, Differential geometric and analytic perspectives. MR:1849187 (2002h:58040)

[8] Shiu Yuen Cheng, Peter Li, and Shing-Tung Yau, Heat equations on minimal submanifolds and their applications, Amer. J. Math. 106 (1984), no. 5, 1033-1065. MR0761578 (85m:58171)

[9] Jaigyoung Choe and Robert Gulliver, Isoperimetric inequalities on minimal submanifolds of space forms, Manuscripta Math. 77 (1992), no. 2-3, 169-189. MR1188579 (93k:53059) 
[10] Doris Fischer-Colbrie and Richard Schoen, The structure of complete stable minimal surfaces in 3-manifolds of nonnegative scalar curvature, Comm. Pure Appl. Math. 33 (1980), no. 2, 199-211. MR0562550 (81i:53044)

[11] Robert Hermann, Focal points of closed submanifolds of Riemannian spaces, Indag. Math. 25 (1963), 613-628. MR0158333 (28:1558)

[12] Shigeo Kawai, Operator $\Delta-a K$ on surfaces, Hokkaido Math. J. 17 (1988), no. 2, 147-150. MR0945852 (89j:58149)

[13] Masatoshi Kokubu, Weierstrass representation for minimal surfaces in hyperbolic space, Tohoku Math. J. (2) 49 (1997), no. 3, 367-377. MR1464184 (98f:53008)

[14] Hiroshi Mori, Remarks on the paper of Barbosa and do Carmo, Arch. Math. (Basel) 37 (1981), no. 2, 173-178. MR0640804 (83e:53061)

[15] Geraldo de Oliveira Filho and Marc Soret, Complete minimal surfaces in hyperbolic space, Math. Ann. 311 (1998), no. 3, 397-419. MR1637915 (2000a:53013)

[16] A. V. Pogorelov, On the stability of minimal surfaces, Dokl. Akad. Nauk SSSR 260 (1981), no. 2, 293-295. MR0630142 (83b:49043)

[17] Konrad Polthier, Geometric a priori estimates for hyperbolic minimal surfaces, Bonner Mathematische Schriften [Bonn Mathematical Publications], 263, Universität Bonn Mathematisches Institut, Bonn, 1994, Dissertation, Universität Bonn, Bonn, 1993. MR:1293964 (95h:53011)

[18] Richard Schoen, Estimates for stable minimal surfaces in three-dimensional manifolds, Seminar on minimal submanifolds, Ann. of Math. Stud., vol. 103, Princeton Univ. Press, Princeton, NJ, 1983, pp. 111-126. MR0795231 (86j:53094)

[19] Karen K. Uhlenbeck, Closed minimal surfaces in hyperbolic 3-manifolds, Seminar on minimal submanifolds, Ann. of Math. Stud., vol. 103, Princeton Univ. Press, Princeton, NJ, 1983, pp. 147-168. MR:0795233 (87b:53093)

Department of Mathematics, California State University, Northridge, Northridge, CALIFORNia 91330

E-mail address: alberto.candel@csun.edu 